\title{
Genetic Control by cis-Acting Regulatory RNAs in Bacillus subtilis: General Principles and Prospects for Discovery
}

\author{
IRNOv, A. KeRTSBURG, AND W.C. WinKLER \\ The University of Texas Southwestern Medical Center, Department of Biochemistry, Dallas, Texas 75390-9038
}

\begin{abstract}
In recent years, Bacillus subtilis, the model organism for gram-positive bacteria, has been a focal point for study of posttranscriptional regulation. In this bacterium, more than 70 regulatory RNAs have been discovered that respond to intracellular proteins, tRNAs, and small-molecule metabolites. In total, these RNA elements are responsible for genetic control of more than $4.1 \%$ of the genome-coding capacity. This pool of RNA-based regulatory elements is now large enough that it has become a worthwhile endeavor to examine their general features and to extrapolate these simple observations to the remaining genome in an effort to predict how many more may remain unidentified. Furthermore, both metabolite- and tRNA-sensing regulatory RNAs are remarkably widespread throughout eubacteria, and it is therefore becoming increasingly clear that some of the observations for $B$. subtilis gene regulation will be generally applicable to many different species.
\end{abstract}

\section{CIS-ACTING REGULATORY RNAS IN THE MODEL BACTERIUM, BACILLUS SUBTILIS}

Microorganisms must be capable of responding to sudden changes in their environment, such as stress conditions and nutrient limitation. To that end, microorganisms use a diverse assortment of genetic strategies for precise coordination of their genes. Although the majority of these mechanisms exert their influence over transcription initiation, a growing number have been found to regulate postinitiation processes. Just how important this posttranscriptional "layer" of gene regulation is to a given bacterium is still an open question. However, studies from $B$. subtilis and Escherichia coli suggest that a substantial portion of the genome is regulated by a combination of cis- and trans-acting regulatory RNAs.

cis-Acting regulatory RNAs are transcribed with the genes they regulate and are the subject of this chapter, whereas trans-acting regulatory RNAs are transcribed separately from their target genes and are discussed elsewhere. Considerable progress has recently been achieved in the study of cis-acting regulatory RNAs, helped considerably by an intensive focus on $B$. subtilis gene regulation. For example, a significant portion $(>4.1 \%)$ of the $B$. subtilis genome has already been postulated to be regulated from the combined contributions of more than 70 cis-acting regulatory RNAs (Winkler 2005). These RNAs can be split into a few different categories: those that respond to intracellular proteins (currently 22 total), tRNAs (19 total), metabolites (currently 27 total), a collection of "orphans" that are likely to respond to metabolites (currently 7 total), and at least half a dozen less-characterized regulatory RNAs (Table 1). The ligand-binding domains for all of these regulatory RNAs are referred to as aptamers, whereas the remaining portions are involved in harnessing ligand-induced conformational changes for genetic control. In general, protein-sensing RNAs regulate a wide variety of genes involved in carbohydrate catabolism, amino acid synthesis, and transport. tRNA-sensing RNAs, called T-box RNAs, regulate aminoacyl tRNA-synthetases, certain amino acid biosynthetic clusters, and transport genes. Metabolite-sensing RNAs primarily control expression of genes responsible for synthesis and transport of cofactors, amino sugars, amino acids, and nucleotides.

An assortment of metabolite-sensing RNAs have been discovered that respond to adenosylcobalamin, thiamine pyrophosphate (TPP), flavin mononucleotide (FMN), guanine, adenine, a precursor for queuine, lysine, glycine, and glucosamine-6-phosphate (GlcN6P). Additionally, three structurally distinct classes that sense $S$-adenosylmethionine (SAM) have been identified (for review, see Grundy and Henkin 2004a; Nudler and Mironov 2004; Winkler and Breaker 2005). With the exception of two SAM-sensing RNA elements, all of these individual classes have been identified in B. subtilis (see Fig. 3). Biochemical analyses suggest that these RNA structures exhibit some preorganization prior to ligand association but are conformationally modified upon binding. In general, gel-based structural probing, equilibrium dialysisbased analyses, and measurements of ligand binding by fluorescence quenching or isothermal titration calorimetry all reveal apparent $K_{\mathrm{D}}$ values that are in the lownanomolar range. Therefore, these natural aptamers bind tightly to their target metabolites. For at least several RNA classes, recent structural data demonstrate how this is achieved (Batey 2006). X-ray crystallographic data reveal that metabolite ligands are selectively stabilized via a combination of specific hydrogen bonding, energy of base stacking, metal ion stabilization, and ionic interactions. 
Table 1. Regulation of B. subtilis Expression by Protein-, tRNA-, and Metabolite-sensing RNAs

\begin{tabular}{l} 
Regulated Gene(s) \\
\hline trpEDCFBA, pabA, trpP, ycbK \\
Tryptophan metabolism \\
glpFK, glpD, glpTO \\
Glycerol metabolism \\
hutPHUIGM \\
Histidine catabolism \\
pyrR, pyrP, pyrB/C/AA/AB/K/D/F/E \\
Pyrimidine synthesis \\
ptsGHI \\
Glucose transport
\end{tabular}

bglPH, $b g l S$

Sugar metabolism (aryl $\beta$-glucoside utilization)

$s a c B, s a c X Y$

Sugar metabolism (sucrose utilization)

$\operatorname{sacP} A$

Sugar metabolism (sucrose utilization)

rho

rpsD

Ribosomal protein $\mathrm{S} 4$

rps $J$

Ribosomal protein $\mathrm{S} 10$

$\operatorname{csp} B, \operatorname{csp} C$

Cold shock response

$\operatorname{pyr} G$

CTP synthesis

cysE-cys $S$

Cysteine synthesis

leuS, pheS-pheT, serS, thrS, thrZ trpS, tyrS, tyrZ, valS, ilvBNCA-leuCBD, rtpA. proB-proA, ileS, proI, glyQ-glyS, alaS hisS-aspS, yvbW

Aminoacyl synthetases, amino acid biosynthesis, and transport yitJ, metI-metC, $y k r T-y k r S, y k r W-y k r X-y k r Y-y k r Z$,

cys $H$-cys $P$-sat-cys $C-y \ln D-y \ln E-y \ln F$, yoaD-yoaCyoaB, metE, metK, yus C-yusB-yusA, yxjG, yxjH

General methionine biosynthesis, methylene tetrahydrofolate reductase, $5^{\prime}$ methylthioadenosine recycling pathway, cysteine biosynthesis, methionine synthase, SAM synthetase, uncharacterized $\mathrm{ABC}$ transporters thiC, tenA1-thiX1-thiY1-thiz1-thiE2-thiO-thiS-thiGthiF-thiD, $y k o F-y k o E-y k o D-y k o C, y u a J, y \operatorname{lm} B$

Thiamine biosynthesis and transport, uncharacterized genes ypaA, ribD-ribE-ribBA-ribH

Riboflavin biosynthesis and flavin transport

yvrC-yvrB-yvrA-yvqK

Unknown; similar to iron transport proteins

lys $C$

Aspartokinase II

yxjA, xpt-pbuX, pbuG, purE-purK-purB-purC-purSpurQ-purL-purF-purM-purN-purH-purD

Purine biosynthesis, pyrimidine nucleoside transport, xanthine permease, hypoxanthine/guanine permease $y d h L$

Uncharacterized transporter

gcv $T / P A / P B$

Glycine efflux

$\operatorname{glm} S$

Glucosamine-6-phosphate synthesis

$y k o K, y k k C D E, y x k D, y d a O, k t r A B, y y b P, y k o Y$

Transport of divalent metal ions, uncharacterized transporters, potassium transporter, unknown genes

\section{Effector(s) ligands and mode of gene control}

Trp-bound TRAP inhibits translation initiation and/or induces transcription termination (for review, see Gollnick et al. 2005).

GlpP protein stabilizes an antiterminator helix (for review, see Stülke 2002).

Histidine-bound HutP protein stabilizes an antiterminator helix (for review, see Kumar et al. 2006).

UMP-bound PyrR protein stabilizes an anti-antiterminator helix (Switzer et al. 1999).

GlcT protein stabilizes an antiterminator helix; GlcT is activated by phosphorylation by HPr and inactivated due to phosphorylation by EIIGlc (for review, see Stülke 2002).

LicT binds to $b g l P H$ and $b g l S$ RNAs and stabilizes an antiterminator helix when cells are exposed to aryl $\beta$-glucosides and are limited for glucose (for review, see Stülke 2002).

In the absence of sucrose, SacY is inactivated through phosphorylation by SacX; in its activated state, SacY stabilizes an antiterminator (for review, see Stülke 2002).

The SacT protein stabilizes an antiterminator helix (for review, see Stülke 2002).

Rho autoregulates itself (Ingham et al. 1999).

S4 is likely to associate to its own UTR for feedback repression (Grundy and Henkin 1992).

The S10 operon is likely to be bound by a ribosomal protein encoded within the operon ( $\mathrm{Li}$ et al. 1997).

$\mathrm{CspB}$ and $\mathrm{CspC}$ promote translation during conditions of low temperature by associating to their 5'UTRs (Graumann and Marahiel 1999).

During conditions of low CTP reiterative transcription adds a polyG sequence that acts to stabilize an antiterminator helix (Meng et al. 2004).

Uncharged tRNA ${ }^{\text {Cys }}$ binds to an intercistronic T-box RNA, instigating antitermination (Pelchat and Lapointe 1999).

Cognate uncharged tRNA binds to the $5^{\prime}$ UTR to promote antitermination (for review, see Grundy and Henkin 2003).

SAM binds to the $5^{\prime}$ UTR to promote termination (for review, see Winkler and Breaker 2005).

TPP binds to the $5^{\prime} \mathrm{UTR}$ to promote termination (for review, see Winkler and Breaker 2005).

FMN binds to the $5^{\prime}$ UTR to promote termination (for review, see Winkler and Breaker 2005).

Adenosylcobalamin binds to the 5'UTR to promote termination (for review, see Winkler and Breaker 2005).

Lysine binds to the $5^{\prime}$ UTR to promote termination (for review, see Winkler and Breaker 2005).

Guanine binds to the $5^{\prime}$ UTR to promote termination (for review, see Winkler and Breaker 2005).

Adenine binds to the $5^{\prime} \mathrm{UTR}$ to promote antitermination (for review, see Winkler and Breaker 2005).

Glycine binds to the 5'UTR to promote antitermination (Mandal et al. 2004).

GlcN6P binds to the 5'UTR to promote autocatalytic selfcleavage, which in turns leads to mRNA destabilization (Winkler et al. 2004).

Unknown metabolite binds to an "orphan" RNA to either promote or disrupt termination formation (Barrick et al. 2004). 


\section{Location}

There are a few examples where $B$. subtilis regulatory RNAs reside within intercistronic regions of an operon. For example, regulatory RNAs that interact with proteins PyrR (pyrimidine-responsive regulator) and HutP (histidine-responsive regulator) are located between these proteins and the rest of the genes in the operon (Oda et al. 1988; Turner et al. 1994). In addition, a tRNA ${ }^{\text {Cys }}$-sensing RNA is found between glt $X$ (encoding glutamyl-tRNA synthetase) and cysE-cysS (encoding serine acetyltransferase and cysteinyl-tRNA synthetase, respectively) and is a site for endoribonuclease cleavage (Gagnon et al. 1994; Pelchat and Lapointe 1999). Similarly, an RNA element that binds to tryptophan-responsive attenuation protein (TRAP) is located within intercistronic spaces between folate synthesis genes $p a b B$ and $p a b A$ as well as between $\operatorname{rtp} A$ and $y c b K$ (Babitzke 2004). However, the majority of $B$. subtilis regulatory RNAs are located within the $5^{\prime}$-untranslated regions (5'UTRs) of specific mRNA transcripts. In contrast, no cis-acting regulatory RNAs have been discovered within the 3'UTR portion.

On rare occasions, regulatory RNAs can occur in tandem. For example, the 5'UTR of B. subtilis thrZ (a cryptic threonyl-tRNA synthetase) contains three consecutive tRNA $^{\text {Thr }}$-sensing RNAs (Putzer et al. 1992). Presumably, this arrangement results in expression of $t h r Z$ only under conditions of extreme threonine starvation or very low levels of threonyl-tRNA synthetase. An interesting variation on this theme is exploited by glycine-sensing RNAs. These RNA structures are composed of two glycine-binding aptamers that exhibit cooperativity for glycine association; glycine binding to the first domain increases glycine binding affinity for the second domain (Mandal et al. 2004). Only upon occupation of both glycine-binding sites is expression of efflux or catabolism genes altered.

\section{Genetic Mechanisms}

In general, bacterial cis-acting regulatory RNAs control gene expression via transcription attenuation or translation inhibition. Both mechanistic strategies rely upon the specific orchestration of alternate base-pairing schemes (Fig. 1). For transcription attenuation, a metabolic signal is received by the aptamer domain that in turn stimulates formation of a transcription termination signal, usually in the form of an intrinsic terminator helix (responsible for rho-independent cessation of transcription). Typically, this occurs by stabilization of an antiantiterminator helical element, thereby allowing terminator formation. It is the interchange between terminator and antiterminator helices that dictate expression levels; therefore, in the absence of the aptamer-bound signal, an alternate antiterminator helix will be formed as the default state configuration. In other instances (e.g., tRNAsensing RNAs) (Grundy and Henkin 2003), the default conformation is formation of a transcription terminator helix. For these RNAs, ligand association promotes formation of an antiterminator helical element, thereby preventing terminator formation, often referred to as transcription antitermination. The strongest experimental

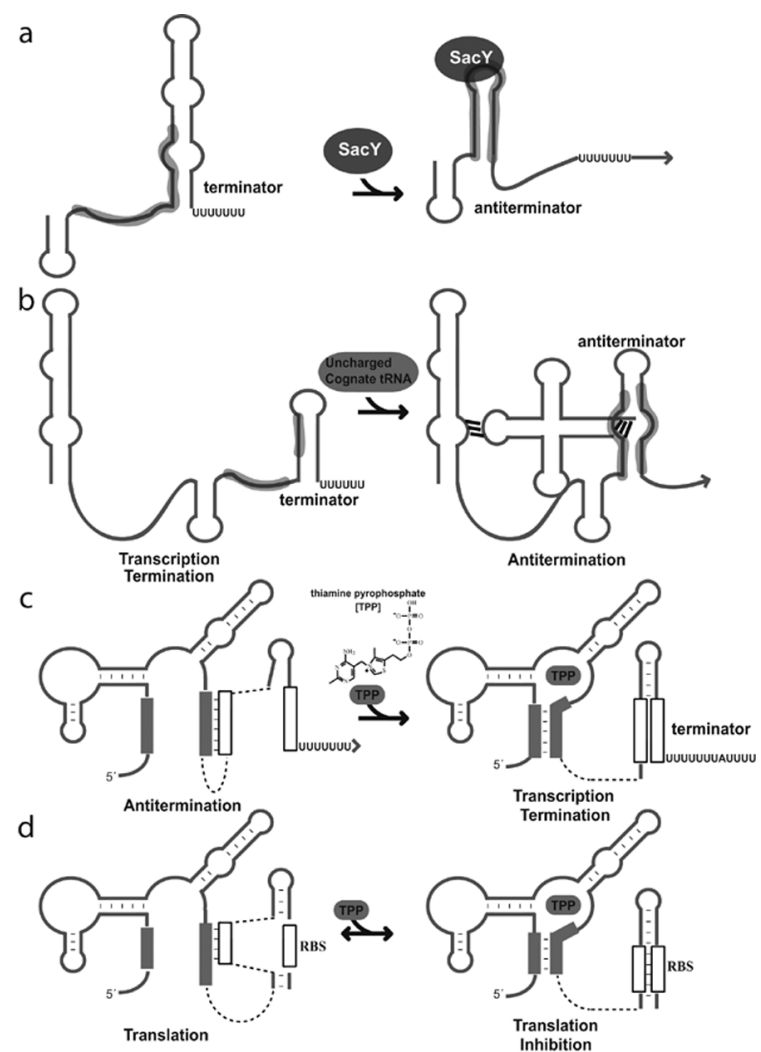

Figure 1. Genetic regulation by cis-acting bacterial regulatory RNAs. (a) Example of a protein-responsive RNA. In the absence of sucrose, SacY is inactivated through phosphorylation by SacX. In its activated state, SacY stabilizes an antiterminator helix for the $s a c B$ and $s a c X Y$ transcripts (for review, see Stülke 2002). (b) Example of tRNA-sensing RNAs (Grundy and Henkin 2003). A decrease in amino acid levels alters intracellular tRNA charging ratios. Uncharged cognate tRNAs associate with the appropriate T-box RNA to promote transcription antitermination, thereby increasing expression of aminoacyl tRNA synthetases, biosynthesis genes, and transporters. (c) Example of a metabolite-sensing regulatory RNA: transcription attenuation. Binding of thiamine pyrophosphate (TPP) stimulates formation of an anti-antiterminator helix, thereby allowing formation of an intrinsic transcription terminator within the 5'UTR of a B. subtilis thiamine biosynthetic cluster (Mironov et al. 2002). (d) Example of a metabolite-sensing regulatory RNA: translation inhibition. Binding of TPP stimulates formation of a helical element that sequesters the ribosome-binding site, thereby reducing translation initiation efficiency for E. coli thiM transcripts (Winkler et al. 2002).

proof for transcription attenuation/antitermination-based mechanisms has derived from their reconstitution in vitro from purified components (RNA polymerase, DNA templates, ribonucleotides, and the appropriate ligand molecule). These latter experiments provide clear evidence for ligand-induced termination in the absence of additional factors, although transcription elongation factors such as NusA may participate in vivo.

A unique feature of these mechanisms is that the outcome is a single "decision" between terminator and antiterminator elements during the active process of transcription. Therefore, the "choice" of forming terminator or antiterminator elements derives from precise coordination of multiple processes including transcription kinet- 
ics, the nascent RNA folding pathway, and ligand-binding kinetics. Indeed, evidence for several different regulatory RNAs suggests that accurate harmonization of transcription and ligand-binding kinetics is important for regulation (Landick et al. 1996; Mironov et al. 2002; Yakhnin and Babitzke 2002; Zhang and Switzer 2003; Grundy and Henkin 2004b; Wickiser et al. 2005). Specifically, by synchronizing RNA polymerases on target DNA templates in vitro and studying transcript products at varying time intervals, one can map profiles for sites of transcriptional pausing and termination. Pausing signals have been found in several instances to be located near sequences required for antiterminator formation. These data suggest that institution of a transcriptional delay while the RNA polymerase footprint overlaps antiterminator or terminator elements may allow time for RNA folding and ligand binding, prior to commitment of the ultimate conformational outcome. Inclusion of NusA into this experimentation alters the profile and strength of transcriptional pausing and termination signals in vitro (Landick et al. 1996; Yakhnin and Babitzke 2002; Zhang and Switzer 2003; Grundy and Henkin 2004b). Therefore, it undoubtedly also affects transcription attenuation/antitermination mechanisms in vivo, further implicating a role for NusA in posttranscriptional processes. For FMN-sensing RNAs, these transcriptional kinetic data have been coupled with measurements of FMN association, which further support this hypothesis. Specifically, an FMN-responsive RNA (B. subtilis ribD) was found to be unable to reach thermodynamic equilibrium with FMN before RNA polymerase could reach the terminator sequence element. Therefore, the kinetics of transcription must be carefully tuned with the rate of FMN association to the aptamer domain. How general all of these observations are for transcription attenuation-based regulatory RNAs remains to be determined.

The second major mode of regulation is through translation inhibition. Similar to transcription attenuation, ligand association influences the thermodynamic interplay between helical pairings. However, rather than controlling terminator formation, the ribosome-binding site (RBS) is either occluded from ribosomal access or rendered more accessible upon ligand binding (Fig. 1) (see, e.g., Nou and Kadner 2000; Schlax and Worhunsky 2003; Winkler et al. 2004; Yakhnin et al. 2004). A combination of genetic and biochemical approaches has been used to demonstrate ligand-induced blocking of ribosomes for the B. subtilis RNAs that exert their regulatory influence via translation inhibition. This experimentation includes $30 \mathrm{~S}$ toeprint analysis, cell-free translation, and structural probing analyses of the regulatory RNA in the presence and absence of the appropriate RNA-binding ligand. Overall, many bacterial regulatory RNAs govern gene expression by affecting the efficiency of translation initiation in this manner. Interestingly, there is a nonrandom phylogenetic distribution for transcription attenuation and translation inhibition mechanisms in eubacterial species. For reasons not yet revealed, gram-positive bacteria preferentially utilize transcription attenuation mechanisms, whereas gram-negative bacteria more often than not rely upon translation inhibition (see, e.g., Rodionov et al.
2002; Vitreschak et al. 2002). For example, more than $90 \%$ of B. subtilis regulatory RNAs rely on transcription attenuation-based mechanisms.

\section{THE GLMS REGULATORY RNA IS A UNIQUE METABOLITE-SENSING RIBOZYME}

As outlined above, there are two predominate methods for harnessing cis-acting regulatory RNAs for bacterial genetic control: transcription attenuation and translation inhibition. However, an alternate mechanism is used by a GlcN6P-sensing RNA identified immediately upstream of the glmS gene (Fig. 2) (Barrick et al. 2004). Synthesis and characterization of the RNA sequence in vitro revealed that GlcN6P promoted an autocatalytic, site-specific cleavage event near the $5^{\prime}$ terminus, thus demonstrating that the RNA sequence is a natural metabolite-responsive ribozyme (Winkler et al. 2004). A reciprocal relationship was observed between ribozyme self-cleavage in vitro and gene expression in vivo. Deleterious site-directed mutations of conserved positions reduced self-cleavage capability in vitro and correspondingly increased expression of a downstream $l a c Z$ reporter in vivo. Therefore, feedback repression of $g l m S$ by intracellular GlcN6P was proposed to be the regulatory function for the ribozyme (Winkler et al. 2004). Indeed, early data indicate that self-cleavage results in release of a 61-nucleotide fragment from the 5' terminus of the approximately $1.9-\mathrm{kb} \mathrm{glmS}$ transcript in response to increasing levels of GlcN6P. This then promotes a significant decrease in intracellular stability for $g \operatorname{lm} S$ transcripts through a process that is dependent on at least one intracellular endoribonuclease (W.C. Winkler, unpubl.). However, the complete mechanism for how selfcleavage imparts genetic control in vivo still remains to be elucidated.

Although study of the $g \operatorname{lm} S$ regulatory pathway in vivo is an emerging subject, biochemical characterization of the self-cleavage reaction in vitro has progressed more rapidly. Addition of GlcN6P has been demonstrated to promote the self-cleavage reaction at least 110,000 -fold over the rate of cleavage in the absence of GlcN6P (McCarthy et al. 2005). The chemical mechanism for self-cleavage appears to be similar to that of other small natural ribozymes, resulting in 5'-hydroxyl and 2'-3'-cyclic phosphate termini (Fig. 2) (Winkler et al. 2004). Recognition of the GlcN6P ligand appears to be highly specific. Even subtle differences in ligand structure, such as removal of the C6-bound phosphate or the sugar amino group, result in dramatic reduction of ligand-induced catalysis (Winkler et al. 2004; McCarthy et al. 2005). A preview into how this ligand recognition is achieved has been recently derived via nucleotide analog interference mapping (NAIM). This method employs backbone and nucleobase analogs to identify RNA functional groups essential for ligand phosphate recognition (Fig. 2) (Jansen et al. 2006). These positions cluster near the site of self-cleavage or between the P1 and P2 helices, all within the minimal core sequence. Additionally, gel-based structural probing techniques argue that the overall RNA structure is significantly preorganized prior to ligand binding and that it is only modestly altered during catalysis (Hampel and Tinsley 2006). 

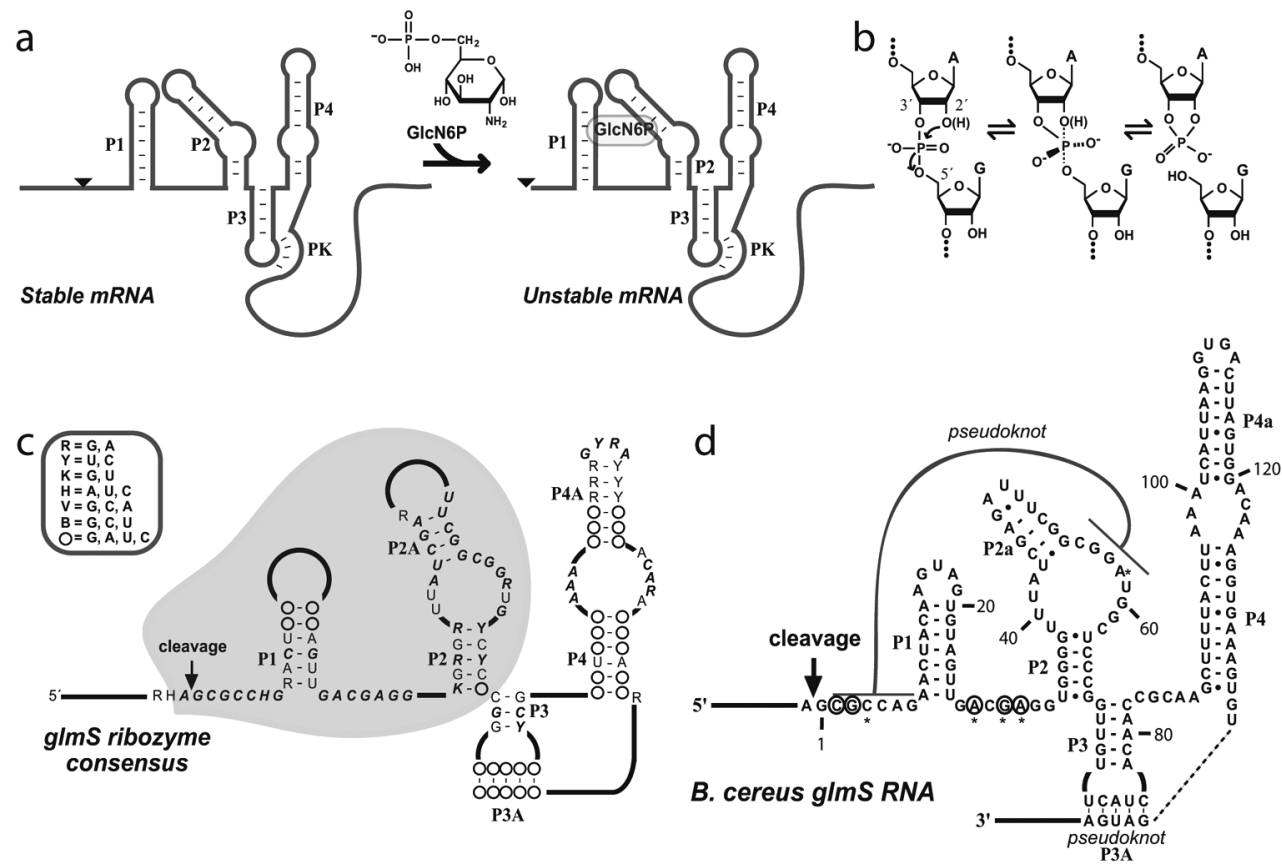

Figure 2. Genetic control by a metabolite-sensing ribozyme. (a) A GlcN6P sensing regulatory RNA is positioned within the $5^{\prime}$ UTR of $g \operatorname{lm} S$ transcripts for many gram-positive bacteria. GlcN6P stimulates an autocatalytic self-cleavage event at the $5^{\prime}$ end of the regulatory RNA, thereby releasing a short oligonucleotide and reducing mRNA stability for downstream glmS transcripts (W.C. Winkler, unpubl.). The details for this mechanism remain to be explored. (b) The proposed chemical mechanism for $g l m S$ ribozyme self-cleavage (Winkler et al. 2004). The $2^{\prime}$ hydroxyl of the -1 position acts as the attacking nucleophile with the bridging $5^{\prime}$ oxygen as the leaving group. The reaction proceeds via an $\mathrm{S}_{\mathrm{N}} 2$-type in-line attack. (c) Consensus secondary structure for glmS ribozymes (Roth et al. 2006). Italicized nucleotides in bold type are conserved in at least $95 \%$ of representative sequences, and non-italicized letters represent greater than $80 \%$ conservation. The site of self-cleavage is indicated with an arrow. (Gray-shaded zone) Minimal ribozyme domain. $(d)$ NAIM experimentation inferred several positions as being the likely sites for magnesium association (black circles) and for recognition of the ligand phosphate (asterisks) (Jansen et al. 2006).

Overall, the ribozyme consists of a series of four conserved stem-loop structures (P1-P4; Winkler et al. 2004) and two functionally important pseudoknot elements (Fig. 2) (Wilkinson and Been 2005; Roth et al. 2006; Soukup 2006). Neither deletion of the P3-P4 helices nor of nucleotides $5^{\prime}$ to the site of cleavage completely eliminates catalysis (Fig. 2) (Winkler et al. 2004). However, cleavage activity of this minimal ribozyme is approximately four orders of magnitude reduced relative to a full-length sequence when supplied with similar magnesium concentrations (Roth et al. 2006). Nonetheless, rate constants for the minimal construct are restored to almost full-length levels upon supplementation with additional magnesium ions ( 100-fold). Therefore, the function of the P3-P4 region is likely to be optimization of $\operatorname{glm} S$ cleavage during physiological magnesium concentrations. However, this requirement for magnesium ions does not necessarily reflect a direct involvement of metal ions in catalysis. Indeed, magnesium could be replaced with cobalt hexammine, a structural mimic of hydrated magnesium, without significant loss in cleavage ability (Roth et al. 2006). Given the very slow rate of solvent exchange for the amine groups of cobalt hexammine, this observation suggests that only outer-shell or electrostatic interactions are required of divalent ions and that they are unlikely to directly participate in catalysis.
A common method for divalent ions to bind RNA is through interactions to nonbridging phosphate oxygens. Single-atom substitution of these positions with sulfur groups is a frequently used method that can be employed to identify these sites of backbone interactions. In many instances where phosphorothioate substitutions instigate a loss of essential magnesium ions, more thiophilic metals such as manganese can functionally substitute the missing divalents. This strategy of phosphorothioate substitution and manganese rescue has recently been employed for $g \operatorname{lmS}$ ribozymes, resulting in the identification of candidate sites for magnesium association, many of which are located near the site of self-cleavage (Fig. 2) (Jansen et al. 2006).

How unusual are ribozymes in biology? To date, only nine distinct classes of naturally occurring RNA catalysts have been identified. There are four small self-cleaving RNAs in addition to $g \operatorname{lm} S$ that all catalyze chain cleavage via an internal transesterification reaction. The hammerhead, hepatitis delta virus, and hairpin ribozymes all assist in processing rolling-circle replication intermediates via self-cleavage (and ligation) activities (Lilley 2003; Fedor and Williamson 2005). The Varkud satellite (VS) RNA is a mitochondrial transcript found in many natural isolates of Neurospora and is also thought to act in the processing of replication intermediates (Lilley 2004). The remaining known catalytic RNAs include RNase P (processing of 
pre-tRNAs), group I and II self-splicing introns, and the ribosome (Lilley 2003; Fedor and Williamson 2005). It remains to be determined whether catalytic RNAs are truly rare in extant organisms, or if their absence is due to a lack of intensive searching. However, the fact that only one category of metabolite-sensing regulatory RNA exploits such a mechanism for regulation suggests that during the course of evolution, transcription attenuation and translation inhibition strategies proved to be more efficient. If so, then why retain self-cleavage ability for the $g \operatorname{lm} S$ RNAs? Three-dimensional resolution of structural features when the RNA is bound to the GlcN6P ligand may eventually provide the answer. In the interim, NAIM analyses have suggested that positions required for ligand phosphate recognition reside in the vicinity of the catalytic site (Jansen et al. 2006). This observation may suggest that GlcN6P binds to preorganized $g l m S$ ribozymes and either participates directly in the chemical mechanism of cleavage or enacts an intimate role in positioning groups at the active site, thereby functioning as a cofactor, rather than an allosteric effector. If this hypothesis proves correct, it may offer an explanation for why, throughout expansive time scales, GlcN6P binding has not been divorced from the self-cleavage reaction and replaced with transcription attenuation- or translation inhibition-based mechanisms.

\section{METABOLITE-SENSING RNAS ARE WIDESPREAD IN BIOLOGY}

One of the most fascinating aspects of metabolite- and tRNA-sensing RNAs is their widespread biological distribution (Fig. 3). In general, metabolite-sensing RNAs are enriched in gram-positive bacteria, although several classes can also be identified in gram-negative species. The metabolite ligands for these RNAs have remained unchanged throughout great evolutionary time scales (White 1976). Therefore, their respective aptamer domains would be expected to have also preserved a common structural fold during the same time period. Indeed, members of a given class of metabolite-sensing RNAs share a common structural architecture, as ascertained by gel-based structural probing and X-ray crystallography (for review, see Winkler and Breaker 2005; Batey 2006). Furthermore, many examples of metabolite-sensing RNAs have been identified in evolutionarily distant organisms. These observations together imply that members of the same RNA class that are discovered in disparate phylogenetic lineages would likely have been present prior to the divergence of the two lineages. Alternatively, the RNA sequences would had to have emerged through convergent evolution, an unlikely explanation given the vastness of potential sequence space and the significant potential for alternate structural solutions (e.g., three structurally distinct SAM sensors have now been identified).

\section{GENERAL FEATURES OF B. SUBTILIS REGULATORY RNAS}

The lengths of B. subtilis regulatory RNAs and the intergenic regions (IGRs) that host them vary among the different RNA classes (Figs. 4 and 5). The average length for protein-, tRNA-, and metabolite-sensing RNAs is approximately 110, 196, and 326 nucleotides in length, respectively (Fig. 4). This trend is also observed for the length of IGRs that harbor these RNAs which, in total, range between 100 and 450 nucleotides in length (Figs. 4 and 5); $90 \%$ of these cis-acting regulatory RNAs reside in IGRs of greater than 200 nucleotides in length. In contrast, more than $70 \%$ of $B$. subtilis IGRs are less than 200 nucleotides and would therefore be unlikely to contain additional regulatory RNAs. Since most of these RNA elements in B. subtilis rely on transcription attenuationbased mechanisms, the region between the end of the regulatory RNA (transcription terminator) and the start of the downstream gene can be quantified. On average, this space is only 50 nucleotides in length, demonstrating that little IGR space is wasted overall. However, it should be pointed out that there is considerable variability in these data (from 1 to 186 nucleotides).

Bacterial genomes range in their nucleotide composition ratios. For example, B. subtilis with a genomic $\mathrm{G}+\mathrm{C}$ average of $43.52 \%$ is considered a "low $\mathrm{G}+\mathrm{C}$ " microorganism, whereas actinomycetes such as Streptomyces coelicolor exhibit substantially higher values. For organisms with reduced $\mathrm{G}+\mathrm{C}$ content, it is possible that intricately structured RNAs may require a higher $\mathrm{G}+\mathrm{C}$ content than the background levels, due to the increased contribution of guanine-cytosine base pairs to overall thermodynamic stability. Therefore, "islands" of higherthan-background $\mathrm{G}+\mathrm{C}$ content may infer IGR locations that contain structured RNAs. The $\mathrm{G}+\mathrm{C} \%$ average for $B$. subtilis total genomic IGRs is 38.1. Indeed, with the exception of protein-sensing RNAs, most of the established cis-acting regulatory RNAs contain regions of increased $\mathrm{G}+\mathrm{C}$ content (50-55\%) (Fig. 5). The fact that this trend differs for protein-sensing RNAs may not be unexpected given that tRNA- and metabolite-sensing RNAs are likely to require more complex structural configurations for construction of their ligand-binding pockets. It is possible that many protein-sensing RNAs rely more on the ability of RNA-binding proteins to either recognize specific short sequences or stabilize certain small secondary structural conformations. Therefore, for some of these RNAs, it may be difficult to define which component is the "ligand" and which may be the "receptor."

An additional measure of RNA stability is its predicted folding energy. It has been observed that highly structured RNAs tend to exhibit lower predicted free energy values as compared to randomized sequence pools (Clote et al. 2005). This is in fact true for all known cis-acting regulatory RNAs in B. subtilis. The predicted folding energy for each of these RNAs is typically three standard deviations lower than that of the average of the folding energy for a pool of 1000 random sequences containing the same dinucleotide frequency (Fig. 5).

Although these observations provide some limited usefulness in the accurate characterization and description of cis-acting regulatory RNAs, it is possible that they could be incorporated with additional criteria for the purpose of predicting locations of novel regulatory RNAs. Importantly, this pool of more than $70 \mathrm{~B}$. subtilis regulatory RNAs does not derive from a comprehensive, sys- 


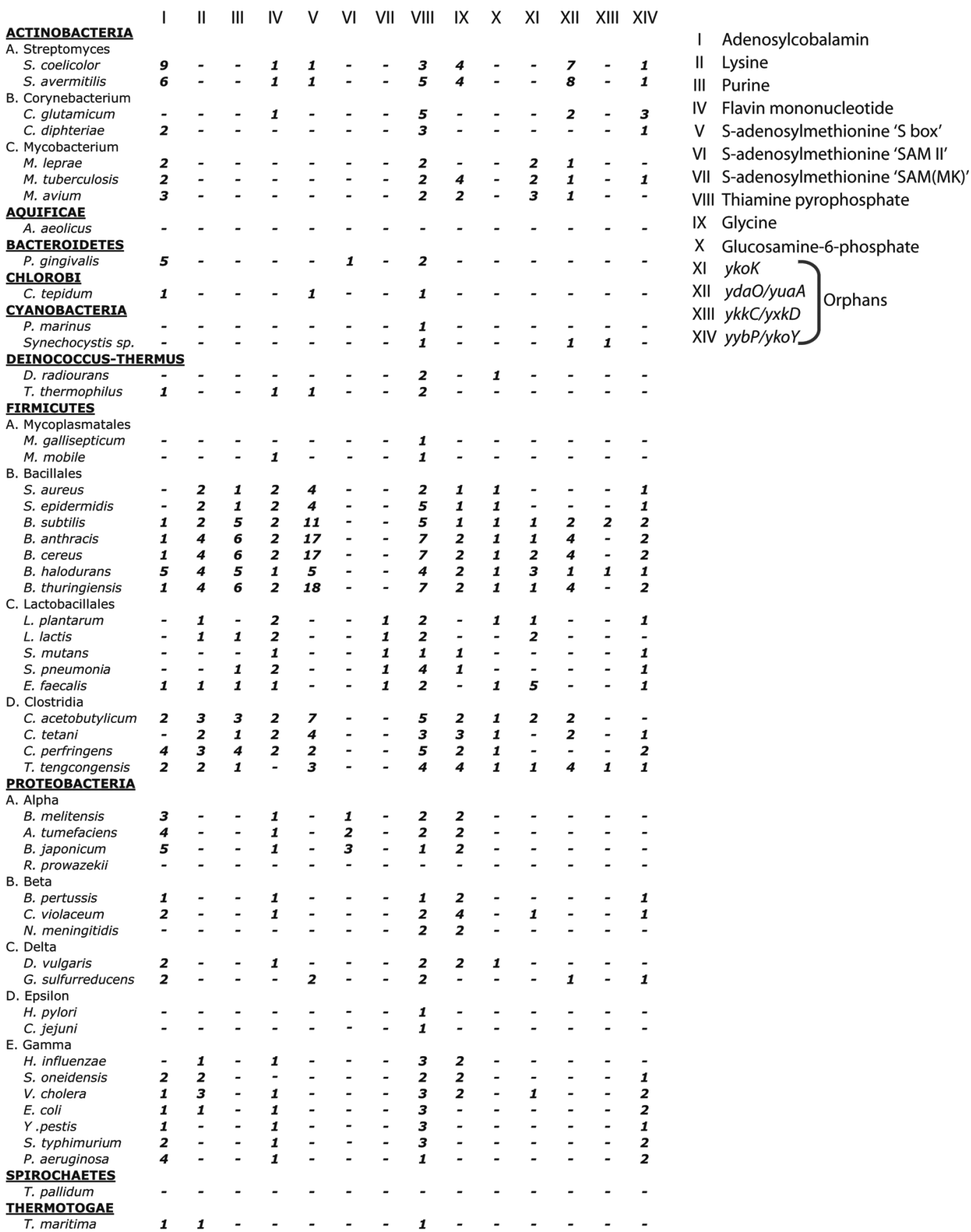

Figure 3. Biological distribution of metabolite-sensing regulatory RNAs. The distribution of different metabolite-sensing RNA classes is shown for representative bacterial species. Data are extracted from RFAM (Griffiths-Jones et al. 2005), except for SAM II and SAM(MK) distribution patterns, which are derived from Corbino et al. (2005) and Fuchs et al. (2006), respectively. Regulatory RNAs that are expected to sense unidentified metabolites ("orphans"; Barrick et al. 2004) are in italics and named after their downstream genes in B. subtilis.

tematic search but from the individual efforts of many investigators spanning several decades. Therefore, this value is still certain to be an underestimate of the true usage for regulatory RNAs in this organism. The genome of B. subtilis contains a total of 7334 IGRs, where an IGR is defined as the nucleotide sequence on a given genomic strand that does not overlap with any genes on the opposite strand of DNA. For this organism, there are 3178
IGRs of suitable length for housing regulatory RNAs (between 100 and 450 nucleotides in length) (Figs. 4 and 5). This represents $43 \%$ of total IGRs and the most optimistic upper limit for the total number of potential regulatory RNAs in this organism, although realistic expectations are certain to be substantially lower. Applying a $\mathrm{G}+\mathrm{C}$ cutoff of more than $48 \%$ would recover virtually all tRNA- and metabolite-sensing RNAs and 
A

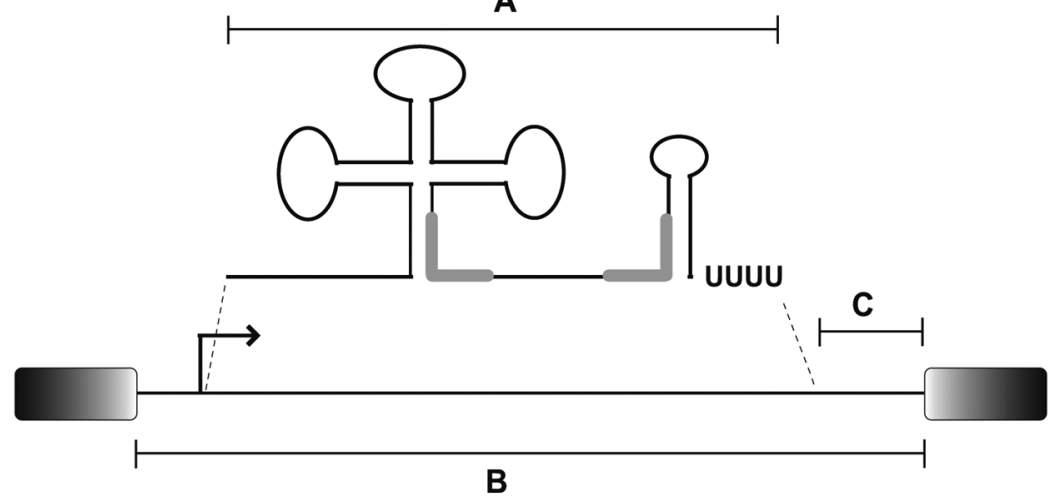

\begin{tabular}{|c|c|c|c|c|c|c|c|c|c|c|c|c|}
\hline \multirow{2}{*}{ Regulatory RNA Class } & \multicolumn{4}{|c|}{ A. Size of regulatory RNA } & \multicolumn{4}{|c|}{ B. Size of IGR } & \multicolumn{4}{|c|}{$\begin{array}{l}\text { C. Distance to downstream } \\
\text { gene }\end{array}$} \\
\hline & Ave. & Stdev. & Max. & Min. & Ave. & Stdev. & Max. & Min. & Ave. & Stdev. & Max. & Min. \\
\hline Protein-sensing RNAs & 109.9 & 27.8 & 151 & 62 & 279.3 & 139.3 & 607 & 124 & 53.9 & 33.1 & 134 & 14 \\
\hline tRNA-sensing RNAs & 326.2 & 161.1 & 813 & 217 & 425.6 & 176.6 & 844 & 248 & 38 & 19.8 & 84 & 16 \\
\hline Metabolite-sensing RNAs & 196.2 & 36.6 & 269 & 107 & 365.8 & 117.6 & 663 & 105 & 54.8 & 44.5 & 186 & 1 \\
\hline All Classes & 204.5 & 120.5 & 813 & 140 & 360.9 & 149.4 & 844 & 105 & 49.8 & 36.8 & 186 & 1 \\
\hline
\end{tabular}

Figure 4. Average sizes for $B$. subtilis regulatory RNAs and the IGR that hosts them. Average lengths from the transcription start to the intrinsic transcription terminator $(A)$, for the entire IGR $(B)$, and from the terminator to the downstream gene $(C)$ are the same as the columns in the table. (Gray boxes) Upstream and downstream genes.

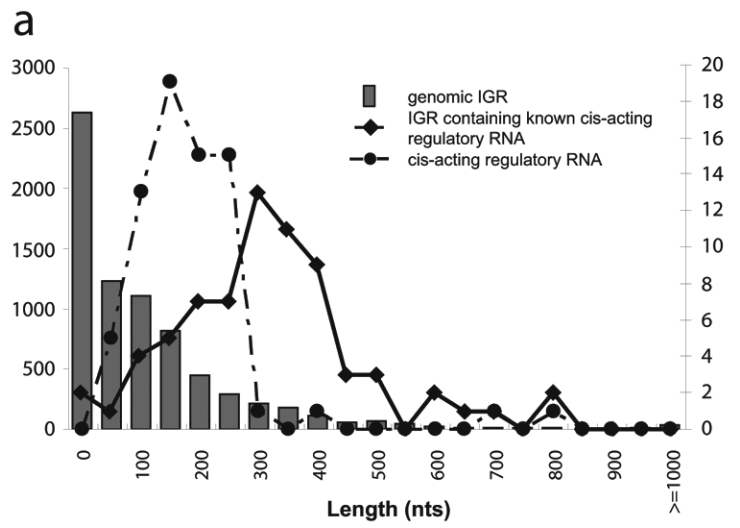

b

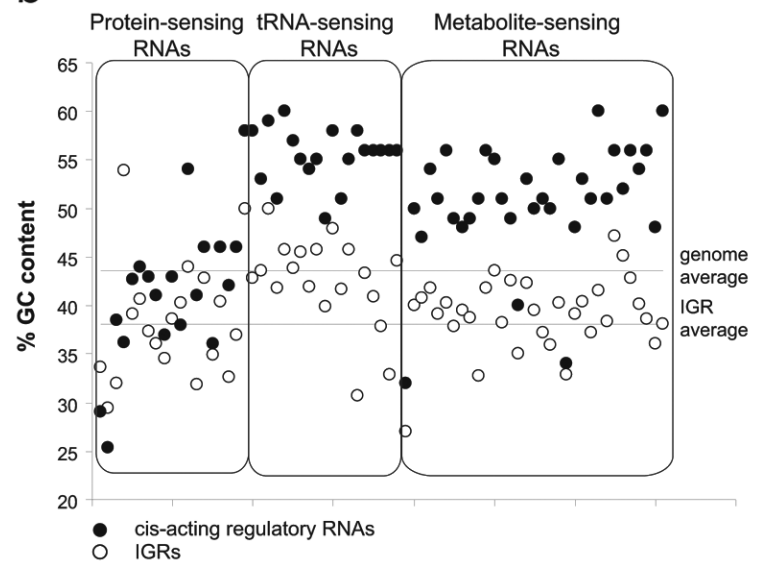

C

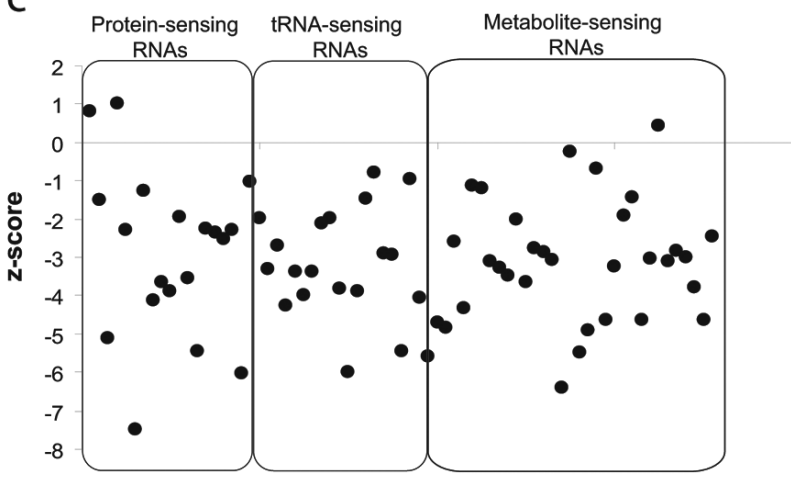

Figure 5. General features of B. subtilis regulatory RNAs. (a) Length distribution of $B$. subtilis regulatory RNAs (circles; right $y$ axis) and the IGRs that host them (diamonds; right $y$ axis). Length distribution for total $B$. subtilis IGRs are indicated by gray-shaded columns (left $y$ axis). (b) Comparison of $\mathrm{G}+\mathrm{C} \%$ content for different classes of $B$. subtilis regulatory RNAs. The $\mathrm{G}+\mathrm{C} \%$ for a sliding window of 100 nucleotides (closed circles) is compared against the $\mathrm{G}+\mathrm{C} \%$ content for the entire IGR that houses known regulatory RNAs (open circles). The horizontal lines indicate the average $\mathrm{G}+\mathrm{C} \%$ values for the total genome $(43.52 \%)$ and for total genomic IGRs (38.1\%). (c) Predicted folding energies for $B$. subtilis regulatory RNAs. Each regulatory RNA sequence was randomly shuffled 1000 times while keeping the same dinucleotide frequency via the Dishuffle program (Clote et al. 2005). A predicted value for free energy of folding was determined via the RNAfold program (Vienna RNA package; Hofacker et al. 1994). The z-score was calculated for each RNA set by comparing the folding free energy of the native sequence to the average of the shuffled pool with respect to standard deviation. 
reduce the total number of candidate IGRs to 454 ( $6 \%$ of total IGRs). This is still almost sixfold greater than the current number of established regulatory RNAs. It is a reasonable hypothesis to expect that there are new regulatory RNAs to be discovered within this IGR pool; however, it is also obvious that additional parameters must be added before simple computational-assisted methods can accurately pinpoint novel RNAs.

Historically, identification of regulatory RNAs has been more accidental than methodical. Most were discovered from genetic and physiology-based study of downstream genes or operons. More recently, researchers have been moving toward more systematic approaches that combine computational methods with experimental verification of candidates. So far, the most successful methods for finding novel, candidate regulatory RNAs have been via comparative sequence analyses. For this, IGRs from a query genomic sequence are compared by BlastN analysis against other genomic sequences, and the resulting hits are manually examined for evidence of primary sequence and secondary structural conservation. Coupled with predictions of gene function for downstream genes, this method has been effective in identifying novel metabolite-sensing RNAs (Barrick et al. 2004; Corbino et al. 2005). The QRNA program is an example of a similar approach that minimizes the manual inspection and has been used for identifying eight new noncoding RNA (ncRNA) candidates in Saccharomyces cerevisiae (McCutcheon and Eddy 2003).

Predictions of thermodynamic stability have been applied as another method for finding regulatory RNAs. As explained in the previous section, this method is typically done by comparing the folding energy of native RNA against pools of randomized sequences (Workman and Krogh 1999; Rivas and Eddy 2000; Clote et al. 2005). RNAz (Washietl et al. 2005) and Dynalign (Uzilov et al. 2006) are both examples of this overall approach. RNAz has been used to predict 78 new ncRNA candidates that are conserved among humans, mice, rats, Fugu, and zebra fish. Dynalign is a sensitive method that can recover almost all Escherichia coli ncRNAs, as well as many unexplored candidates.

Candidate regulatory RNAs can also be identified by locating genetic elements that are common in either transcription attenuation or translation inhibition mechanisms. Specifically, using algorithms for identification of promoter and terminator elements, trans-acting regulatory RNAs have been identified in E. coli (Argaman et al. 2001). Additionally, the prediction of terminator and antiterminator pairings within $B$. subtilis IGRs proved to be a relatively successful approach for identification of established regulatory RNAs, as well as the prediction of numerous additional candidates (Merino and Yanofsky 2005).

Due to a high background of false positives, however, these purely computational approaches are still likely to be limited in their usefulness when applied in the absence of additional parameters. These methods and others may be bolstered in the near future by the addition of different transcriptome-based analyses. The latter methods are currently used primarily for genome annotation and expres- sion profiling (Ruan et al. 2004). However, tag-based methods for mapping of exact $5^{\prime}$ and $3^{\prime}$ termini, as well as whole-genome tiling microarrays, may prove to be useful approaches for identifying candidate mRNAs with long UTRs, excellent starting points for discovery of regulatory RNAs (Bertone et al. 2004; Harbers and Carninci 2005). Coupling of such long UTR data sets with the other above-mentioned computational methods may, over time, prove to be the most comprehensive and accurate approach for regulatory RNA identification.

\section{CONCLUSIONS}

Regulatory RNAs convey a simple method of coupling metabolic sensing to gene control. This paper has provided a general summary of the different classes of regulatory RNAs in B. subtilis. This pool now consists of greater than 70 examples of protein-, tRNA-, and metabolite-sensing RNAs. Therefore, RNA-mediated genetic control can no longer be viewed as gene regulatory anomalies, given their extensive use in organisms such as B. subtilis. Although the list of RNAs in this bacterium is not complete, it is large enough so that it can be examined for general features. In the near future, these basic features may prove to be useful in deciding on criteria that could assist in the computational identification of novel regulatory RNAs. In general, establishment of a comprehensive catalog of $B$. subtilis cis-acting regulatory RNAs will help elucidate the contribution of posttranscriptional regulation to overall bacterial genetic regulation.

\section{Note Added in Proof}

During preparation of this manuscript, an example of a B. subtilis protein that binds a 3'UTR for apparent control of mRNA stability was reported by Serio et al. (2006). This discovery further expands the overall $B$. subtilis catalog of regulatory RNAs and hints at a novel 3'UTRbased mechanism.

\section{ACKNOWLEDGMENTS}

W.C.W. acknowledges the financial support provided by the Searle Scholars Program and the University of Texas Southwestern Medical Center Endowed Scholar Program.

\section{REFERENCES}

Argaman L., Hershberg R., Vogel J., Bejerano G., Wagner E.G., Margalit H., and Altuvia S. 2001. Novel small RNA-encoding genes in the intergenic regions of Escherichia coli. Curr. Biol. 11: 941 .

Babitzke P. 2004. Regulation of transcription attenuation and translation initiation by allosteric control of an RNA-binding protein: The Bacillus subtilis TRAP protein. Curr. Opin. Microbiol. 7: 132.

Barrick J.E., Corbino K.A., Winkler W.C., Nahvi A., Mandal M., Collins J., Lee M., Roth A., Sudarsan N., Jona I., et al. 2004. New RNA motifs suggest an expanded scope for riboswicthes in bacterial genetic control. Proc. Natl. Acad. Sci. 101: 6421.

Batey R.T. 2006. Structures of regulatory elements in mRNAs. Curr. Opin. Struct. Biol. 16: 299. 
Bertone P., Stolc V., Royce T.E., Rozowsky J.S., Urban A.E., Zhu X., Rinn J.L., Tongprasit W., Samanta M., Weissman S., et al. 2004. Global identification of human transcribed sequences with genome tiling arrays. Science 306: 2242.

Clote P., Ferre F., Kranakis E., and Krizanc D. 2005. Structural RNA has lower folding energy than random RNA of the same dinucleotide frequency. RNA 11: 578 .

Corbino K.A., Barrick J.E., Lim J., Welz R., Tucker B.J., Puskarz I., Mandal M., Rudnick N.D., and Breaker R.R. 2005. Evidence for a second class of S-adenosylmethionine riboswitches and other regulatory RNA motifs in alpha-proteobacteria. Genome Biol. 6: R70.

Fedor M.J. and Williamson J.R. 2005. The catalytic diversity of RNAs. Nat. Rev. Mol. Cell Biol. 6: 399.

Fuchs R.T., Grundy F.J., and Henkin T.M. 2006. The $\mathrm{S}_{\mathrm{MK}}$ box is a new SAM-binding RNA for translational regulation of SAM synthetase. Nat. Struct. Mol. Biol. 13: 226.

Gagnon Y., Breton R., Putzer H., Pelchat M., Grunberg-Manago M., and Lapointe J. 1994. Clustering and co-transcription of the Bacillus subtilis genes encoding the aminoacyl-tRNA synthetases specific for glutamate and for cysteine and the first enzyme for cysteine biosynthesis. J. Biol. Chem. 269: 7473.

Gollnick P., Babitzke P., Antson A., and Yanofsky C. 2005. Complexity in regulation of tryptophan biosynthesis in Bacillus subtilis. Annu. Rev. Genet. 39: 47.

Graumann P. and Marahiel M.A. 1999. Cold shock proteins $\mathrm{CspB}$ and $\mathrm{CspC}$ are major stationary-phase-induced proteins in Bacillus subtilis. Arch. Microbiol. 171: 135.

Griffiths-Jones S., Moxon S., Marshall M., Khanna A., Eddy S.R., and Bateman A. 2005. Rfam: Annotating non-coding RNAs in complete genomes. Nucleic Acids Res. 33: D121.

Grundy F.J. and Henkin T.M. 1992. Characterization of the Bacillus subtilis rpsD regulatory target site. J. Bacteriol. 174: 6763.

. 2003. The $\mathrm{T}$ box and $\mathrm{S}$ box transcription termination control systems. Front. Biosci. 8: d20.

. 2004a. Regulation of gene expression by effectors that bind to RNA. Curr. Opin. Microbiol. 7: 126.

. 2004b. Kinetic analysis of tRNA-directed transcription antitermination of the Bacillus subtilis glyQS gene in vitro. J. Bacteriol. 186: 5392.

Hampel K.J. and Tinsley M.M. 2006. Evidence for preorganization of the $\operatorname{glm} S$ ribozyme ligand binding pocket. Biochemistry 45: 7861.

Harbers M. and Carninci P. 2005. Tag-based approaches for transcriptome research and genome annotation. Nat. Methods 2: 495 .

Hofacker I.L., Fontana W., Stadler P.F., Bonhoeffer S., Tacker M., and Schuster P. 1994. Fast folding and comparison of RNA secondary structures. Monatsh. Chem. 125: 167.

Ingham C.J., Dennis J., and Furneaux P.A. 1999. Autogenous regulation of transcription termination factor Rho and the requirement for Nus factors in Bacillus subtilis. Mol. Microbiol. 31: 651.

Jansen J.A., McCarthy T.J., Soukup G.A., and Soukup J.K. 2006. Backbone and nucleobase contacts to glucosamine-6phosphate in the glmS ribozyme. Nat. Struct. Mol. Biol. 13: 517.

Kumar P.K., Kumarevel T., and Mizuno H. 2006. Structural basis of HutP-mediated transcription anti-termination. Curr. Opin. Struct. Biol. 16: 18.

Landick R., Turnbough C.L., and Yanofsky C. 1996. Transcription attenuation. In Escherichia coli and Salmonella: Cellular and molecular biology (F.C. Neidhardt et al.), p. 1263. ASM Press, Washington, D.C.

Li X., Lindahl L., Sha Y., and Zengel J.M. 1997. Analysis of the Bacillus subtilis $\mathrm{S} 10$ ribosomal protein gene cluster identifies two promoters that may be responsible for transcription of the entire 15-kilobase S10-spc-alpha cluster. J. Bacteriol. 179: 7046.

Lilley D.M. 2003. The origins of RNA catalysis in ribozymes. Trends Biochem. Sci. 28: 495.

2004. The Varkud satellite ribozyme. RNA 10: 151.

Mandal M., Lee M., Barrick J.E., Weinberg Z., Emilsson G.M.,
Ruzzo W.L., and Breaker R.R. 2004. A glycine-dependent riboswitch that uses cooperative binding to control gene expression. Science 306: 275.

McCutcheon J.P. and Eddy S.R. 2003. Computational identification of non-coding RNAs in Saccharomyces cerevisiae by comparative genomics. Nucleic Acids Res. 31: 4119.

McCarthy T.J., Plog M.A., Floy S.A., Jansen J.A., Soukup J.K., and Soukup G.A. 2005. Ligand requirements for $\mathrm{glmS}$ ribozyme self-cleavage. Chem. Biol. 12: 1221.

Meng Q., Turnbough C.L., Jr., and Switzer R.L. 2004. Attenuation control of pyrG expression in Bacillus subtilis is mediated by CTP-sensitive reiterative transcription. Proc. Natl. Acad. Sci. 101: 10943.

Merino E. and Yanofsky C. 2005. Transciption attenuation: A highly conserved regulatory strategy used by bacteria. Trends Genet. 21: 260.

Mironov A.S., Gusarov I., Rafikov R., Lopez L.E., Shatalin K., Kreneva R.A., Perumov D.A., and Nudler E. 2002. Sensing small molecules by nascent RNA: A mechanism to control transcription in bacteria. Cell 111: 747 .

Nou X. and Kadner R.J. 2000. Adenosylcobalamin inhibits ribosome binding to btuB mRNA. Proc. Natl. Acad. Sci. 97: 7190.

Nudler E. and Mironov A.S. 2004. The riboswitch control of bacterial metabolism. Trends Biochem. Sci. 29: 11.

Oda M., Sugishita A., and Furukawa K. 1988. Cloning and nucleotide sequences of histidase and regulatory genes in Bacillus subtilis hut operon and positive regulation of the operon. J. Bacteriol. 170: 3199.

Pelchat M. and Lapointe J. 1999. In vivo and in vitro processing of the Bacillus subtilis transcript coding for glutamyl-tRNA synthetase, serine acetyltransferase, and cysteinyl-tRNA synthetase. RNA 5: 281.

Putzer H., Gendron N., and Grunberg-Manago M. 1992. Coordinate expression of the two threonyl-tRNA synthetase genes in Bacillus subtilis: Control by transcriptional antitermination involving a conserved regulatory sequence. $E M B O$ J. 11: 3117 .

Rivas E. and Eddy S.R. 2000. Secondary structure alone is generally not statistically significant for the detection of noncoding RNAs. Bioinformatics 16: 583.

Rodionov D.A., Vitreschak A.G., Mironov A.A., and Gelfand M.S. 2002. Comparative genomics of thiamin biosynthesis in procaryotes. New genes and regulatory mechanisms. J. Biol. Chem. 277: 48949.

Roth A., Nahvi A., Lee M., Jona I., and Breaker R.R. 2006. Characteristics of the $g \operatorname{lm} S$ ribozyme suggest only structural roles for divalent metal ions. RNA 12: 607 .

Ruan Y., Le Ber P., Ng H.H., and Liu E.T. 2004. Interrogating the transcriptome. Trends Biotechnol. 22: 23.

Schlax P.J. and Worhunsky D.J. 2003. Translational repression mechanisms in prokaryotes. Mol. Microbiol. 48: 1157.

Serio A.W., Pechter K.B., and Sonenshein A.L. 2006. Bacillus subtilis aconitase is required for efficient late-sporulation gene expression. J. Bacteriol. 188: 6396.

Soukup G.A. 2006. Core requirements for $g \operatorname{lm} S$ ribozyme selfcleavage reveal a putative pseudoknot structure. Nucleic Acids Res. 34: 968.

Stülke J. 2002. Control of transcription termination in bacteria by RNA-binding proteins that modulate RNA structures. Arch. Microbiol. 177: 433.

Switzer R.L., Turner R.J., and Lu Y. 1999. Regulation of the Bacillus subtilis pyrimidine biosynthetic operon by transcriptional attenuation: Control of gene expression by an mRNA-binding protein. Prog. Nucleic Acid Res. Mol. Biol. 62: 329 .

Turner R.J., Lu Y., and Switzer R.L. 1994. Regulation of the Bacillus subtilis pyrimidine biosynthetic (pyr) gene cluster by an autogenous transcriptional attenuation mechanism. $J$. Bacteriol. 176: 3708.

Uzilov A.V., Keegan J.M., and Matthes D.H. 2006. Detection of non-coding RNAs on the basis of predicted secondary structure formation free energy change. BMC Bioinformatics 7: 173.

Vitreschak A.G., Rodionov D.A., Mironov A.A., and Gelfand M.S. 2002. Regulation of riboflavin biosynthesis and trans- 
port genes in bacteria by transcriptional and translational attenuation. Nucleic Acids Res. 30: 3141.

Washietl S., Hofacker I.L., and Stadler P.F. 2005. Fast and reliable prediction of noncoding RNAs. Proc. Natl. Acad. Sci. 102: 2454

White H.B. 1976. Coenzymes as fossils of an earlier metabolic state. J. Mol. Evol. 7: 101.

Wickiser J.K., Winkler W.C., Breaker R.R., and Crothers D.M. 2005. The speed of RNA transcription and metabolite binding kinetics operate an FMN riboswitch. Mol. Cell 18: 49.

Wilkinson S.R. and Been M.D. 2005. A pseudoknot in the 3' non-core region of the $\mathrm{glm} S$ ribozyme enhances self-cleavage activity. RNA 11: 1788 .

Winkler W.C. 2005. Metabolic monitoring by bacterial mRNAs. Arch. Microbiol. 183: 151.

Winkler W.C. and Breaker R.R. 2005. Regulation of bacterial gene expression by riboswitches. Annu. Rev. Microbiol. 59: 487.

Winkler W., Nahvi A., and Breaker R.R. 2002. Thiamine deriva- tives bind messenger RNAs directly to regulate bacterial gene expression. Nature 419: 952.

Winkler W.C., Nahvi A., Roth A., Collins J.A., and Breaker R.R. 2004. Control of gene expression by a natural metaboliteresponsive ribozyme. Nature 428: 281.

Workman C. and Krogh A. 1999. No evidence that mRNA have lower folding free energies than random sequences with the same dinucleotide distribution. Nucleic Acids Res. 27: 4816.

Yakhnin A.V. and Babitzke P. 2002. NusA-stimulated RNA polymerase pausing and termination participates in the Bacillus subtilis trp operon attenuation mechanism in vitro. Proc. Natl. Acad. Sci. 99: 11067.

Yakhnin H., Zhang H., Yakhnin A.V., and Babitzke P. 2004. The trp RNA-binding attenuation protein of Bacillus subtilis regulates translation of the tryptophan transport gene $\operatorname{trp} P$ (yhaG) by blocking ribosome binding. J. Bacteriol. 186: 278 .

Zhang H. and Switzer R.L. 2003. Transcriptional pausing in the Bacillus subtilis pyr operon in vitro: A role in transcriptional attenuation? J. Bacteriol. 185: 4764. 


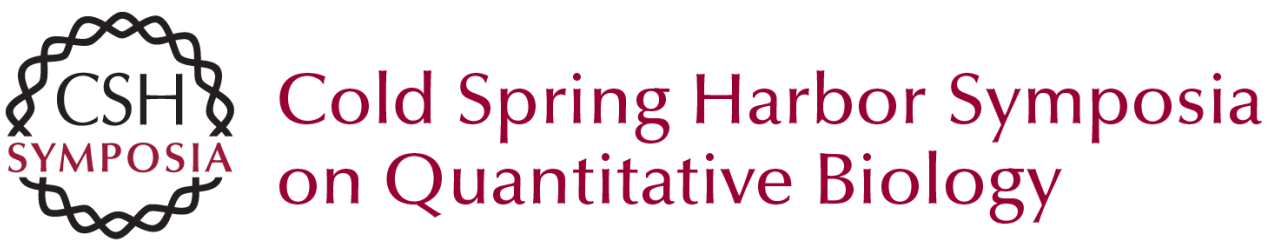

\section{Genetic Control by cis-Acting Regulatory RNAs in Bacillus subtilis : General Principles and Prospects for Discovery}

IRNOV, A. KERTSBURG and W.C. WINKLER

Cold Spring Harb Symp Quant Biol 2006 71: 239-249

Access the most recent version at doi:10.1101/sqb.2006.71.021

References This article cites 61 articles, 21 of which can be accessed free at: http://symposium.cshlp.org/content/71/239.full.html\#ref-list-1

License

Email Alerting

Receive free email alerts when new articles cite this article - sign up in Service the box at the top right corner of the article or click here. 